\title{
Licochalcone-E induces caspase-dependent death of human pharyngeal squamous carcinoma cells through the extrinsic and intrinsic apoptotic signaling pathways
}

\author{
SANG-JOUN YU ${ }^{1}$, IN-A CHO ${ }^{2}, \mathrm{KYEONG-ROK} \mathrm{KANG}^{2}, \mathrm{YI}^{-R A} \mathrm{JUNG}^{3}$, SEUNG SIK CHO ${ }^{4}$, \\ GOO YOON $^{4}$, JI-SU OH ${ }^{5}$, JAE-SEEK YOU ${ }^{5}$, YO-SEOB SEO ${ }^{6}$, GYEONG-JE LEE ${ }^{7}$, SOOK-YOUNG LEE ${ }^{3}$, \\ DO KYUNG KIM ${ }^{8}$, CHUN SUNG KIM ${ }^{8}$, SU-GWAN KIM ${ }^{3,5,8}$, MI-AE JEONG ${ }^{9}$ and JAE-SUNG KIM ${ }^{10}$ \\ ${ }^{1}$ Department of Periodontology, School of Dentistry; ${ }^{2}$ Department of Biodental Engineering, Graduate School; \\ ${ }^{3}$ Regional Innovation Center for Dental Science and Engineering, Chosun University, Gwangju 501-759; \\ ${ }^{4}$ Department of Pharmacy, College of Pharmacy, Mokpo National University, Mokpo, Jeollanamdo 353-729; \\ Departments of ${ }^{5}$ Oral and Maxillofacial Surgery, ${ }^{6}$ Oral and Maxillofacial Radiology and ${ }^{7}$ Prosthodontics; \\ ${ }^{8}$ Oral Biology Research Institute, Chosun University, Gwangju 501-759; ${ }^{9}$ Department of \\ Dental Hygiene, Kangwon National University, Samcheok, Gangwon 259-13; ${ }^{10}$ Pre-Dentistry, \\ School of Dentistry, Chosun University, Gwangju 501-759, Republic of Korea
}

Received August 4, 2015; Accepted February 3, 2017

DOI: $10.3892 / 01.2017 .5865$

\begin{abstract}
The aim of the present study was to investigate licochalcone-E (Lico-E)-induced apoptosis and the associated apoptotic signaling pathway in FaDu cells, a human pharyngeal squamous carcinoma cell line. Treatment with Lico-E exhibited significant cytotoxicity on FaDu cells in a concentration-dependent manner. The $\mathrm{IC}_{50}$ value of Lico-E in FaDu cells was $\sim 50 \mu \mathrm{M}$. Treatment with Lico-E increased the number of dead FaDu cells. Furthermore, chromatin condensation, which is associated with apoptotic cell death, was observed in $\mathrm{FaDu}$ cells treated with Lico-E for $24 \mathrm{~h}$. By contrast, Lico-E did not produce cytotoxicity or increase the number of dead cells when applied to human normal oral keratinocytes (hNOKs). Furthermore, chromatin condensation was not observed in hNOKs treated with Lico-E. Treatment with Lico-E increased the expression of Fas ligand and the cleaved form of caspase-8 in FaDu cells. Furthermore, treatment with
\end{abstract}

Correspondence to: Professor Jae-Sung Kim, Pre-Dentistry, School of Dentistry, Chosun University, 309 Philmun Boulevard, Dong, Gwangju 501-759, Republic of Korea

E-mail: js_kim@chosun.ac.kr

Abbreviations: Apaf-1, apoptotic protease-activating factor 1; Bad, Bcl-2-associated agonist of cell death; Bax, apoptosis regulator $\mathrm{BAX}$; Bcl-2, apoptosis regulator Bcl-2; Bcl-xL, Bcl-2-like protein 1; FasL, Fas ligand; hNOKs, human normal oral keratinocytes; Lico-E, licochalcone-E; OC, oral cancer; OD, optical density; PARP, poly (ADP-ribose) polymerase; SD, standard deviation

Key words: licochalcone-E, apoptosis, human carcinoma cells, caspases, chemotherapeutic agent
Lico-E increased the expression of pro-apoptotic factors, including apoptosis regulator BAX, Bcl-2-associated agonist of cell death, apoptotic protease-activating factor 1, caspase-9 and tumor suppressor p53, while decreasing the expression of anti-apoptotic factors, including apoptosis regulator Bcl-2 and Bcl-2-like protein 1 in FaDu cells. The expression of cleaved caspases-3 and poly (ADP-ribose) polymerase was significantly upregulated following treatment with Lico-E in FaDu cells, while Lico-E-induced apoptotic FaDu cell death was partially suppressed by treatment with Z-VAD-FMK, a pan caspase inhibitor. Therefore, Lico-E-induced oral cancer (OC) cell-specific apoptosis is mediated by the death receptor-dependent extrinsic and mitochondrial-dependent intrinsic apoptotic signaling pathways. In conclusion, these data suggested that Lico-E exhibits potential chemopreventive effects and warrants further developed as a chemotherapeutic agent against $\mathrm{OC}$.

\section{Introduction}

Oral cancer (OC) is one of the most common cancers, comprising $\sim 5 \%$ of all annual cancer diagnoses worldwide (1). Previous studies reported that the occurrence of OC is associated with alcohol consumption $(2,3)$, smoking $(3,4)$, betel nut chewing (5), and human papilloma virus infection (6). Despite improved clinical interventions, including surgery, radiotherapy, chemotherapy and chemo-radiotherapy, the 5-year survival rate for patients with OC has remained poor over the previous several decades (7). Typically, OC includes types of cancer formed on the lips, oral cavity and oropharynx (8). Clinical interventions for patients with OC may produce alterations in appearance or oral function (9). Previously, to reduce side effects and to synergistically enhance radiotherapeutic efficacy in patients with OC, 
chemotherapy has been considered to be a clinical strategy for the treatment of OC (10). Although chemotherapy has beneficial effects, including the induction of cancer cell death, and the retardation of cancer cell migration or metastasis, it has several side effects including high toxicity to normal cells and low efficacy in cancer cells, and it may lead to drug resistance (10).

Previous studies on the development of chemotherapeutic agents have been focused on inducing cancer-specific cell death through the modulation of apoptosis, or programmed cell death. Furthermore, to overcome the side effects of current chemotherapeutic agents, studies have focused on the anti-cancer activity of natural compounds purified from herbal plants (7,11-15). As a result of these studies, the US Food and Drug Administration approved the clinical use of a medicinal herbal plant-derived natural compound with anti-cancer activity for cancer therapy (16).

Licorice, the common name for roots from plants of the Glycyrrhiza genus, including Glycyrrhiza glabra, Glycyrrhiza inflate, and Glycyrrhiza uralensis, is a herbal plant extract used in traditional folk medicine in Asia. Licorice has been used as a medication for stomach ulcers, bronchitis and sore throats $(7,17)$. Furthermore, the anticancer activity of licorice has been reported in breast $(18,19)$, oral $(11)$, colon (12) and prostate cancer (13). Licorice inhibits metastasis (18), has anti-proliferative properties (19), increases apoptosis and may cause cell cycle arrest (14). Recently, the authors demonstrated that licochalcone-A, a natural phenolic chalconoid purified from licorice (15), induces caspase-dependent apoptotic FaDu cell death through the extrinsic and intrinsic apoptotic signaling pathways.

Lico-E has recently been isolated from the roots of Glycyrrhiza species (20) and has been demonstrated to possess anti-inflammatory properties (21), antimicrobial activity (22), antioxidant activity (23), antidiabetic effects (24) and anticancer properties (25). However, the biological functions of Lico-E have not been completely examined.

Therefore, the present study aimed to determine whether Lico-E functions as a chemotherapeutic agent against OC. Furthermore, the potential apoptotic effect of Lico-E on OC was evaluated and the associated apoptotic signaling pathway was elucidated.

\section{Materials and methods}

Preparation of Lico-E. The Glycyrrhiza roots were purchased from the Chonnam Herb Association (Gwangju, Korea). A voucher specimen (MNUYG-003) was deposited at the College of Pharmacy, Mokpo National University (Mokpo, Korea). The air-dried, powdered Glycyrrhiza species roots $(600 \mathrm{~g})$ were extracted twice with 4 liter $100 \%$ methanol using sonication for $3 \mathrm{~h}$. Following filtration with filter paper (Advantec, Osaka, Japan), the methanol extract was evaporated and suspended in distilled water and then defatted with 1 liter n-hexane. The aqueous layer was partitioned with methylene chloride ( $3 \times 1$ liter). The evaporation residue ( $5 \mathrm{~g}$ ) was subjected to flash silica gel chromatography, using an n-hexane:ethyl acetate:methanol solvent system (2:1:0.1, 1.5:1:10.1, 1:1:0.1 and $100 \%$ methanol), to afford 10 fractions. Fractions were subjected to further flash silica gel chromatography, with a chloroform:methanol (100:1) eluent system, to afford Lico-E (5 mg). Lico-E was further purified by column chromatography using RP18 (YMC Co., Ltd., Kyoto, Japan) to an analytically acceptable purity.

Cell culture. Normal human oral keratinocytes (hNOKs) were purchased from ScienCell Research Laboratories, Inc. (Carlsbad, CA, USA). The hNOKs were maintained in Dulbecco's modified Eagle's medium (Thermo Fisher Scientific, Inc., Waltham, MA, USA) containing $10 \%$ fetal bovine serum (FBS; Thermo Fisher Scientific, Inc.).

$\mathrm{FaDu}$, a human pharyngeal squamous carcinoma cell line, was obtained from the American Type Culture Collection (Manassas, VA, USA) and cultured according to the protocol provided. FaDu cells were maintained in minimum essential medium (Thermo Fisher Scientific, Inc.) containing 10\% FBS. Cells were grown in a humidified incubator at $37^{\circ} \mathrm{C}$ containing $5 \% \mathrm{CO}_{2}$.

Cell viability assay. FaDu cells and hNOKs were seeded at a density of $5 \times 10^{5}$ cells $/ \mathrm{ml}$ in 96 -well plates, and allowed to attach to the well overnight. Following incubation, the cultured cells were treated with $12.5,25$ or $50 \mu \mathrm{M}$ Lico-E in triplicate, and incubated at $37^{\circ} \mathrm{C}$ for 24 h. $20 \mu \mathrm{l}$ of $5 \mathrm{mg} / \mathrm{ml}$ MTT was subsequently added to each well and cells were incubated for an additional $4 \mathrm{~h}$ at $37^{\circ} \mathrm{C}$. In order to dissolve the resulting formazan, the cells were resuspended in $200 \mu 1$ dimethyl sulfoxide, and the optical density (OD) of the solution was determined using a spectrometer at an incident wavelength of $570 \mathrm{~nm}$. The experiments were repeated three times, independently. The mean OD \pm standard deviation (SD) for each group of replicates was calculated. The inhibitory rate of cell growth was calculated using the following equation: $\%$ growth inhibition $=[(1-\mathrm{OD}$ extract treated $) /(\mathrm{OD}$ negative control $)] \times 100$.

Cell survival assay. Cell survival was measured, as previously described (7), using calcein-AM to stain the live cells and ethidium bromide homodimer 1 to stain the dead cells. These reagents were obtained from Molecular Probes (Eugene, OR, USA). For the cell survival assay, FaDu cells and hNOKs were plated at a density of $2 \times 10^{4}$ cells in an 8 -well chamber slide, incubated with $12.5,25$ or $50 \mu \mathrm{M}$ Lico-E for $24 \mathrm{~h}$, and subsequently stained with green calcein-AM and ethidium homodimer-1 for $30 \mathrm{~min}$ at room temperature, according to the manufacturer's protocol. The cells were observed and images were captured using inverted phase contrast microscopy (Eclipse TE2000; Nikon Corporation, Tokyo, Japan).

Nucleus staining using DAPI. FaDu cells and hNOKs that had been treated with Lico-E and incubated for $24 \mathrm{~h}$ were fixed with $4 \%$ paraformaldehyde at $4^{\circ} \mathrm{C}$ for $10 \mathrm{~min}$, prior to washing with PBS. The washed cells were stained with $1 \mathrm{mg} / \mathrm{ml}$ DAPI; Roche Diagnostics (Basel, Switzerland) for $20 \mathrm{~min}$. Nuclear condensation was observed by fluorescence microscopy (Eclipse TE2000).

Western blot analysis. FaDu cells (density of $5 \times 10^{6}$ cells $/ \mathrm{ml}$ ) were plated on culture dishes and incubated for $24 \mathrm{~h}$ in a humidified incubator at $37^{\circ} \mathrm{C}$. Cultured FaDu cells were treated 
with Lico-E for $24 \mathrm{~h}$. Cells were harvested, lysed using a cell lysis buffer (cat no. \#9803; Cell Signaling Technology, Danvers, MA, USA) containing protease and phosphatase inhibitor cocktails, and incubated for $1 \mathrm{~h}$ at $4^{\circ} \mathrm{C}$. Lysates were centrifuged at $14,000 \times \mathrm{g}$ for $10 \mathrm{~min}$ at $4^{\circ} \mathrm{C}$. The supernatant was used as the cytosolic fraction. Total protein concentrations of the cell lysates were determined by bicinchoninic acid protein assays (Thermo Fisher Scientific, Inc.). Loading buffer (5X; \#S2002; Biosesang, Sungnam, Korea) was added to equal amounts $(40 \mu \mathrm{g})$ of protein and the mixture was boiled at $90^{\circ} \mathrm{C}$ for $10 \mathrm{~min}$. Total proteins were separated using $10 \%$ SDS-PAGE and transferred to nitrocellulose membranes. Following blocking for $2 \mathrm{~h}$ with $5 \%$ bovine serum albumin (BSA; Sigma-Aldrich; Merck KGaA) in Tris-buffered saline with Tween-20 (TBST) at room temperature, membranes were incubated with primary antibody at $4^{\circ} \mathrm{C}$ overnight and then incubated with horseradish peroxidase-conjugated secondary antibody (dilution in TBST with 5\% BSA, 1:20,000; \#31,460; Thermo Fisher Scientific, Inc.) at room temperature for $2 \mathrm{~h}$. The antibodies used to study the apoptotic signaling pathways included antibodies against Fas ligand (FasL; 40 kDa; \#4273), cleaved caspase-3 (17 and 19 kDa; \#9661), cleaved caspase-8 (18 kDa, \#9496), cleaved caspase-9 (37 kDa; \#7237), poly(ADP-ribose) polymerase (PARP; preform, $116 \mathrm{kDa}$ and cleaved form, $85 \mathrm{kDa}$; \#9542), p53 (53 kDa; \#2527), apoptosis regulator $\mathrm{Bcl}-2$ (Bcl-2; $26 \mathrm{kDa}$;3498), Bcl-2-like protein 1 (Bcl-xL; 30 kDa; \#2762), Bcl-2 associated X (Bax; 20 kDa; \#2772), Bcl-2-associated agonist of cell death (Bad; $23 \mathrm{kDa}$, \#9292), apoptotic protease-activating factor 1 (Apaf-1; $135 \mathrm{kDa}, \# 87,233)$ and $\beta$-actin (45 kDa, \#4970), which were purchased from Cell signaling Technology, Inc. (Danvers, MA, USA) and diluted to 1:1,000 in TBST with 5\% BSA for western blotting. The immunoreactive bands were visualized using an Enhanced Chemiluminescent System (GE Healthcare Life Sciences, Chalfont, UK) and were exposed on radiographic film.

Caspase-3/-7 activity assay. The apoptotic activity of the executioner caspases 3 and 7 was determined using the cell-permeable fluorogenic substrate, PhiPhiLux- $\mathrm{G}_{1} \mathrm{D}_{2}($ OncoImmunin, Inc., Gaithersburg, MD, USA), according to the manufacturer's protocol.

Caspase-dependent cell survival assay. Cells were plated at a density of $1 \times 10^{5}$ cells $/ \mathrm{ml}$ in 96 -well plates and allowed to adhere for $24 \mathrm{~h}$ in a humidified incubator at $37^{\circ} \mathrm{C}$. Following incubation, cultured cancer cells were treated with Lico-E in the presence or absence of $50 \mu \mathrm{M}$ caspase-3 inhibitor (Z-VAD-FMK or N-Benzyloxycarbonyl-Val-Ala-Asp (O-Me) fluoromethyl ketone; Sigma-Aldrich; Merck KGaA, Darmstadt, Germany) and were incubated for $24 \mathrm{~h}$ at $37^{\circ} \mathrm{C}$. Following incubation, cytotoxicity was measured with an MTT assay as previously described.

Statistical analysis. Statistical analysis of the data was performed using SPSS version 19 (SPSS, Chicago, IL, USA). Values are presented as the mean \pm SD of three independent experiments performed in triplicate. Statistical analysis was performed using the Student's t-test. $\mathrm{P}<0.05$ was considered to indicate a statistically significant difference.

\section{Results}

Treatment with Lico-E increases the cytotoxicity of FaDu cells. Incubation of FaDu and hNOK cells with Lico-E for $24 \mathrm{~h}$, followed by the MTT assay to measure cytotoxicity, demonstrated that exposure to $12.5,25$ or $50 \mu \mathrm{M}$ Lico-E did not affect the cell viability of hNOK cells, which were used as a model of normal cells (Fig. 2A). By contrast, cytotoxicity was increased to $17.98 \pm 4.1(\mathrm{P}<0.01)$ and $54.9 \pm(\mathrm{P}<0.01)$ for FaDu cells treated with 25 and $50 \mu \mathrm{M}$ Lico-E compared to an untreated control, respectively (Fig. 2B). The $\mathrm{IC}_{50}$ value of Lico-E was $\sim 50 \mu \mathrm{M}$ for FaDu cells. These results demonstrate that Lico-E is cytotoxic to FaDu cells without affecting the viability of the normal hNOKs.

Lico-E induces FaDu cell death. To verify the Lico-E-induced cytotoxicity, FaDu cells and hNOKs were treated with Lico-E for $24 \mathrm{~h}$, and live cell and dead cell assays were performed. As demonstrated in Fig. 3A, few dead cells were observed when hNOKs were treated with $12.5,25$ or $50 \mu \mathrm{M}$ Lico-E for $24 \mathrm{~h}$. By contrast, the number of dead FaDu cells was increased following treatment with Lico-E. These results are consistent with the hypothesis that Lico-E induces FaDu cell death through cytotoxicity without affecting normal cells.

Lico-E-induced FaDu cell death is mediated by apoptosis. Chromatin condensation is a representative feature of apoptosis. To determine whether Lico-E-induced cell death involves apoptosis, DAPI staining of cultured cells treated with Lico-E for $24 \mathrm{~h}$ was performed to observe the morphology of the nucleus. As demonstrated in Fig. 3B, nuclear condensation was not observed when hNOKs were treated with Lico-E. When FaDu cells were treated with Lico-E, the number of cells with morphological alteration of the nucleus or a condensed nucleus increased significantly. These data indicate that Lico-E-induced FaDu cell death is mediated by apoptosis.

Lico-E-induced apoptosis is mediated by death receptor-dependent extrinsic and mitochondrial-dependent intrinsic apoptotic signaling pathways. As demonstrated in Fig. 4A, the expression of FasL (48 kDa), a representative death receptor ligand and trigger of the apoptotic signaling pathway, was increased by Lico-E in FaDu cells in a concentrationdependent manner. Sequentially, expression of cleaved caspase-8 (18 kDa), a downstream target molecule associated with the death receptor-dependent apoptotic signaling pathway, was increased in the FaDu cells treated with Lico-E in a concentration-dependent manner. Furthermore, the expression of Bcl-2 (26 kDa) and Bcl-xL (26 kDa), anti-apoptotic factors associated with the mitochondrial-dependent intrinsic apoptotic signaling pathway, was decreased in the FaDu cells treated with Lico-E, as demonstrated in Fig. 4B. In addition, the expression of Bax (26 kDa), Bad (23 kDa), Apaf-1 (130 kDa) and cleaved caspase-9 (37 kDa), pro-apoptotic factors associated with the mitochondrial-dependent intrinsic apoptotic signaling pathway, was increased by treatment with Lico-E in FaDu cells in a concentration-dependent manner. Furthermore, the expression of tumor suppressor p53 was increased in the FaDu cells treated with Lico-E. The expression of cleaved caspase-3 (17 and $19 \mathrm{kDa}$ ), a downstream target molecule of 


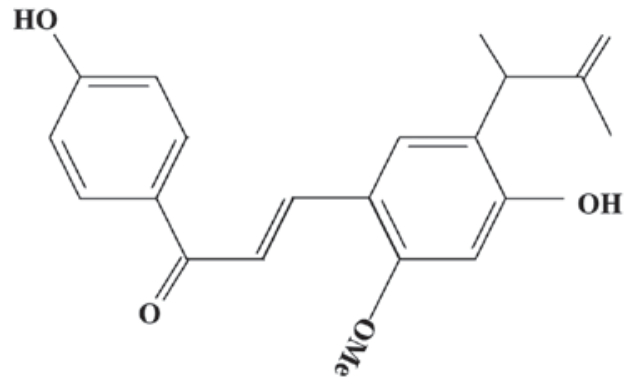

Figure 1. Chemical structure of licochalcone-E.
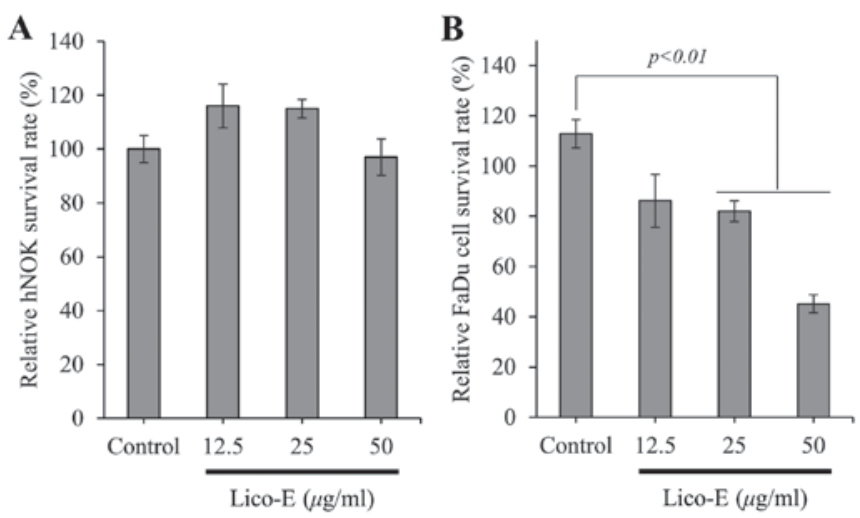

Figure 2. Treatment with Lico-E (A) does not affect hNOK survival but (B) decreases FaDu cell survival. Lico-E, licochalcone-E; hNOK, human normal keratinocyte.

caspase- 8 and -9 , and PARP (89 kDa), a downstream target molecule of caspase-3, was increased in the FaDu cells treated with Lico-E (Fig. 4C). Therefore, these results demonstrate that Lico-E-induced apoptosis of FaDu cell is mediated by the death receptor-dependent extrinsic and mitochondrial-dependent intrinsic apoptotic signaling pathways.

Lico-E-induced apoptosis requires the activation of caspases in FaDu cells. To confirm the activation of caspase-3 in $\mathrm{FaDu}$ cells treated with Lico-E, the caspase-3/-7 activity assay was performed using the cell-permeable fluorogenic substrate PhiPhiLux- $G_{1} D_{2}$. The cleavage of PhiPhiLux $-G_{1} D_{2}$ by activated caspase- 3 was significantly upregulated in $\mathrm{FaDu}$ (Fig. 5A) cells treated with 25 and $50 \mu \mathrm{M}$ Lico-E. Furthermore, the pan caspase inhibitor Z-VAD-FMK partially inhibited Lico-E-induced FaDu cytotoxicity (Fig. 5B). Furthermore, the activation of caspase-3 and its downstream target molecule PARP in the FaDu cells treated with Lico-E was suppressed partially by Z-VAD-FMK (Fig. 5C). These data suggest that Lico-E-induced apoptosis depends upon the activation of caspases in FaDu cells.

\section{Discussion}

Although clinical cancer interventions including surgery, radiotherapy and chemotherapy have advanced rapidly, the 5 -year survival rate of patients with OC has not increased over the previous decade (26). In particular, surgical treatment of OC may lead to more severe side effects, including
$\mathbf{A}$

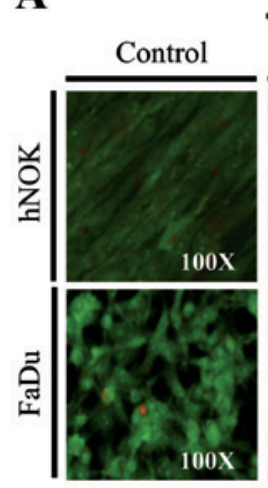

B
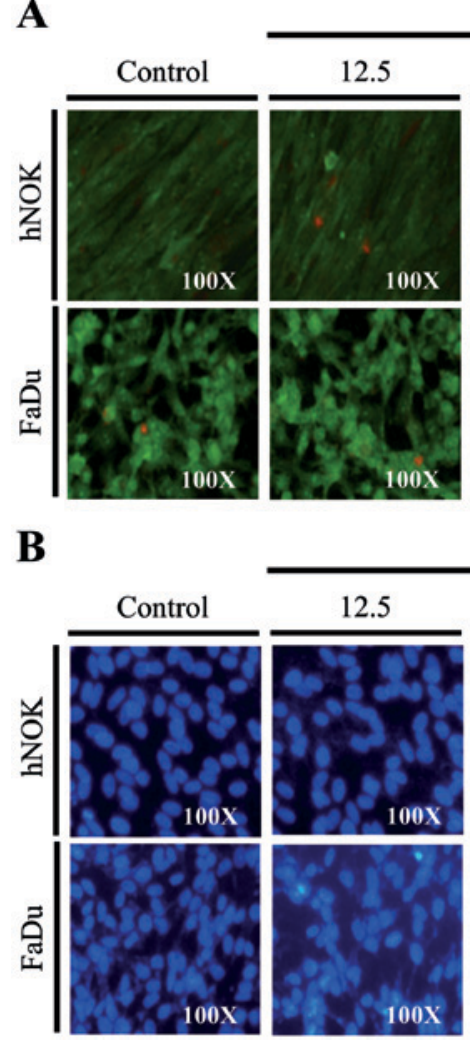

Lico-E $(\mu \mathrm{g} / \mathrm{ml})$

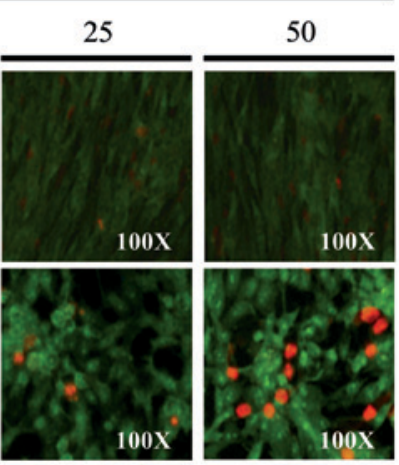

Figure 3. Lico-E induces FaDu cell death through apoptosis. (A) Live cells were stained with green calcein-AM and dead cells were stained with ethidium homodier-1 (red). (B) DAPI staining was performed to observe chromatin condensation. Lico-E, licochalcone-E; hNOK, human normal keratinocyte.

oral cavity dysfunction, and aesthetic and psychological problems, compared with the surgical treatment of other types of cancer (27). Therefore, to reduce the risk of side effects from OC surgery, the tumor size is required to be reduced by chemotherapy or radiotherapy, prior to surgical intervention. Therefore, chemotherapy has been considered to be the primary clinical treatment of OC.

Current clinical chemotherapeutic agents have adverse side effects, including a low efficacy to produce cancer-specific cell death, high toxicity to normal cells, anorexia, nausea and vomiting (28). Therefore, there is a need for chemotherapeutic agents with increased levels of biological safety and efficacy for producing cancer-specific cell death. The development of chemotherapeutic agents for cancer have been focused on the anticancer activities of natural compounds isolated from herbal plants that have had their biological safety verified in folk and traditional medicine (29).

Notably, the roots of licorice (Glycyrrhiza) have been used to treat inflammation, gastric ulcers and atherosclerosis, in folk and traditional herbal medicine in Eastern Asian countries, including Korea, Japan and China (30,31). Licorice root contains alkaloids, polysaccharides, and flavonoids (32). Previous studies have reported licorice-induced cancer-specific cell death in various cancer cell types, including oral $(7,11)$, prostate (13), colon (33) and breast cancer $(34,35)$, through the inhibition of proliferation (11) and metastasis (18), cell-cycle arrest $(13,14)$, and apoptotic cell death (14). Licochalcone-A, a chalconoid and a type of natural phenol, has been isolated 

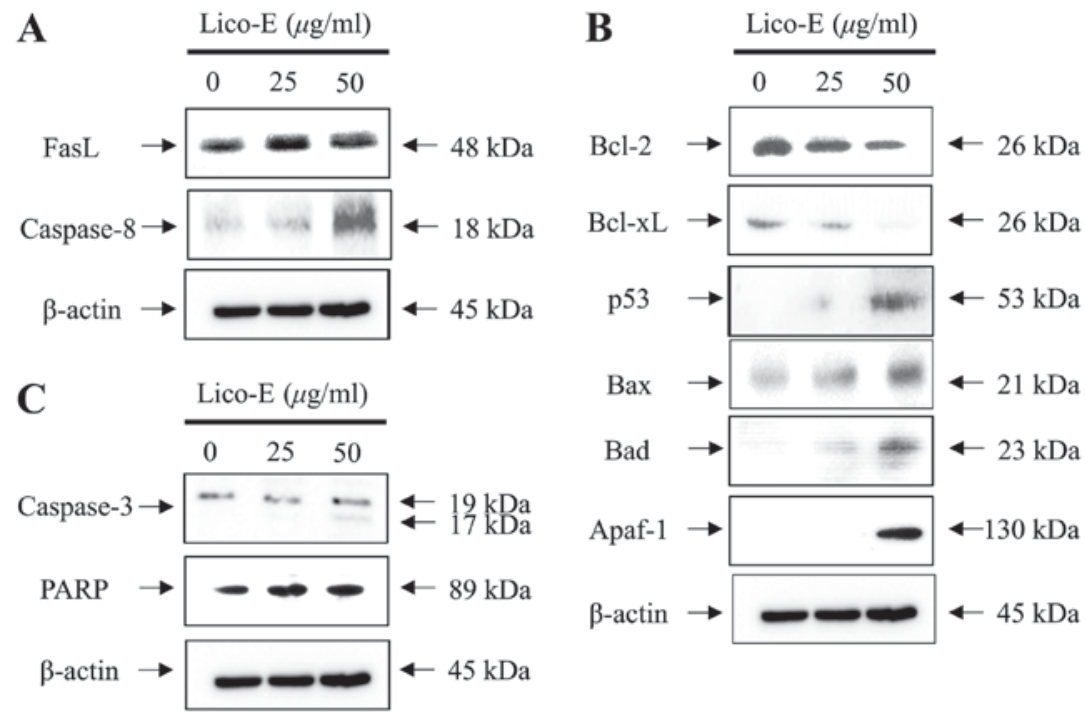

Figure 4. Lico-E-induced apoptosis is mediated by the death receptor-dependent extrinsic and mitochondrial-dependent intrinsic apoptotic signaling pathways in FaDu cells. (A) Western blotting of FasL and caspase-8. (B) Western blotting of Bcl-2, Bcl-xL, p53, Bax, Bad and Apaf-1. (C) Western blotting of caspase-3 and PARP. Lico-E, licochalcone-E; FasL, Fas ligand; Bcl-2, apoptosis regulator Bcl-2; Bcl-xL, Bcl-2-like protein 1; Bax, apoptosis regulator BAX; Bad, Bcl-2-associated agonist of cell death; Apaf-1, apoptotic protease-activating factor 1; PARP, poly (ADP-ribose) polymerase.
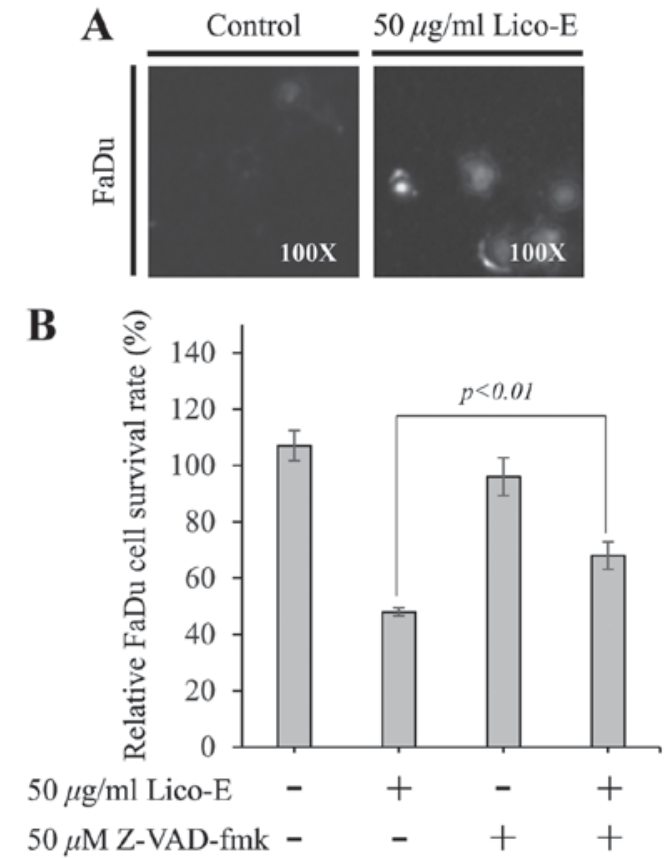

C

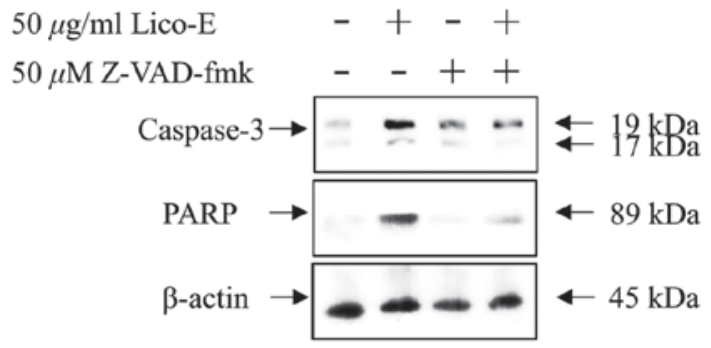

Figure 5. Lico-E-induced apoptosis is dependent on the activation of caspases in FaDu cells. (A) PhiPhiLux-caspase-3/-7 substrate was cleaved in $\mathrm{FaDu}$ cells treated with Lico-E. (B) Z-VAD-FMK, a pan-caspase inhibitor, partially reversed Lico-E-induced cell death in FaDu cells. (C) Activation of caspase-3 and PARP in and FaDu cells treated with Lico-E was inhibited in the presence of Z-VAD-FMK. Lico-E, licochalcone-E; PARP, poly (ADP-ribose) polymerase. from the root of the licorice plant and has exhibited various pharmacological effects, including antimalarial, anticancer, antibacterial and antiviral properties (7). Furthermore, treatment with licochalcone-A induced apoptosis in various cancer cell types, including oral (36), bladder (37,38), lung (39), gastric (40) and prostate cancer cells (41). In addition, Lico-E, a retrochalone, with various pharmacological effects, including antiparasitic, antibacterial, antioxidative and superoxide-scavenging properties, has been isolated from the root of licorice (24). Although Kwon et al (25) reported that Lico-E suppressed lung metastasis in 4T1 mammary orthotopic cancer, Lico-E-induced anticancer properties remain unknown in OC. Therefore, in the present study it was demonstrated that treatment with Lico-E induced apoptotic cell death in OC cells, including head and neck squamous carcinoma FaDu cells, compared with hNOK cells used as normal cells.

The biological safety of Lico-E was demonstrated through the measurement of cytotoxicity in the hNOKs, which were used as a normal. Chang et al (42) have reported that Lico-E induced cell death in ECV 302 cells, which are an immortalized human umbilical vein endothelial cell line. However, in the present study, Lico-E did not affect the cytotoxicity of the primary cultured hNOKs, whereas cytotoxicity was increased in the FaDu cells treated with Lico-E. Consistent with these results, the number of dead cells was increased in the FaDu cells treated with Lico-E. Furthermore, Lico-E did not increase the number of dead cells in the hNOKs. These results indicate that Lico-E induces OC death and may have a lower side effect spectrum compared with other chemotherapeutic agents.

To induce cancer-specific cell death, many studies have focused on the modulation of the apoptotic signaling pathway (10). In the present study, the apoptosis of FaDu cells treated with Lico-E was observed. Chromatin condensation is indicative of apoptosis (43). Chromatin condensation was significantly increased in the FaDu cells treated with Lico-E. Chromatin condensation was not observed when hNOK cells were treated with Lico-E. Therefore, these data suggest that 
Lico-E-induced FaDu cell death is mediated by apoptosis and that Lico-E may modulate the apoptotic signaling pathway. The apoptotic signaling pathway is primarily classified as the death receptor-dependent extrinsic apoptotic signaling pathway or the mitochondrial-dependent intrinsic signaling pathway. The death receptor-dependent extrinsic apoptotic signaling pathway is activated by the binding of expressed death ligands, including FasL (44) and tumor necrosis factor-related apoptosis-inducing ligand (45), with the death receptors on the cell surface. Subsequently, Fas-associated protein with death domain, a Fas receptor adaptor molecule, initiates cell death through the cleavage of caspase-8, caspase- 3 and PARP in sequence (46). Cleaved caspase- 8 of the death receptor-dependent extrinsic apoptotic pathway initiates the mitochondrial-dependent intrinsic apoptotic signaling pathway through the cleavage of cytosolic BH3-interacting domain death agonist (BID) to truncated BID (47). Truncated BID promotes the loss of mitochondrial transmembrane potential through insertion of Bax into the outer mitochondrial membrane (47). Simultaneously, anti-apoptotic factors, including $\mathrm{Bcl}-2$ and $\mathrm{Bcl}-\mathrm{xL}$ are downregulated, while pro-apoptotic factors, including Bax and Bad, are upregulated and initiate the cleavage of caspase-9 (48). Cleaved caspase-9 induces cell death through the cleavage of caspase- 3 and PARP in the mitochondrial-dependent intrinsic apoptotic pathway. Modulation of apoptosis and its signaling pathways have emerged as a critical target for developing chemotherapeutic agents based on natural compounds isolated from medicinal herbal plants (7). Previously, Kwon et al (25) have reported that Lico-E induces apoptosis through the upregulation of Bax and cleaved caspase- 3 and the downregulation of Bcl-2 in tumor tissues of animals xenografted with 4T1 mammary carcinoma cells. In the present study, treatment with Lico-E increased the expression of the death receptor ligand FasL in FaDu cells in a concentration-dependent manner. Subsequently, expressed FasL triggered the death receptor-dependent extrinsic and mitochondrial-dependent intrinsic apoptotic signaling pathways in the FaDu cells treated with Lico-E. Furthermore, apoptosis depends on the activation of caspases, including caspase-8, -7, -9 and -3. In the present study, it was demonstrated that the pan-caspase inhibitor Z-VAD-FMK partially suppressed Lico-E-induced apoptosis, through the inhibition of caspase cleavage in FaDu cells. The results of the present study suggest that Lico-E-induced cell death in FaDu cells is dependent on the activation of caspases involved in the intrinsic and extrinsic apoptotic pathways triggered by FasL expression.

In conclusion, the results of the present study suggest that Lico-E, a potential therapeutic compound derived from natural herbal plants, may be used in the clinical chemotherapy of OC.

\section{Acknowledgements}

The present study was supported by the 2016 research fund from Chosun University Dental Hospital (Gwangju, Korea).

\section{References}

1. Sturgis EM and Miller RH: Second primary malignancies in the head and neck cancer patient. Ann Otol Rhinol Laryngol 104: 946-954, 1995
2. Rothman KJ: The effect of alcohol consumption on risk of cancer of the head and neck. Laryngoscope 88 (1 Pt 2 Suppl 8): S51-S55, 1978.

3. Marron M, Boffetta P, Zhang ZF, Zaridze D, Wünsch-Filho V, Winn DM, Wei Q, Talamini R, Szeszenia-Dabrowska N, Sturgis EM, et al: Cessation of alcohol drinking, tobacco smoking and the reversal of head and neck cancer risk. Int J Epidemiol 39: 182-196, 2010.

4. Hashibe M, Hunt J, Wei M, Buys S, Gren L and Lee YC: Tobacco, alcohol, body mass index, physical activity, and the risk of head and neck cancer in the prostate, lung, colorectal, and ovarian (PLCO) cohort. Head Neck 35: 914-922, 2013.

5. Lin YS, Jen YM, Wang BB, Lee JC and Kang BH: Epidemiology of oral cavity cancer in taiwan with emphasis on the role of betel nut chewing. ORL J Otorhinolaryngol Relat Spec 67: 230-236, 2005.

6. Smith EM, Rubenstein LM, Haugen TH, Pawlita M and Turek LP: Complex etiology underlies risk and survival in head and neck cancer human papillomavirus, tobacco, and alcohol: A case for multifactor disease. J Oncol 2012: 571862, 2012.

7. Park MR, Kim SG, Cho IA, Oh D, Kang KR, Lee SY, Moon SM, Cho SS, Yoon G, Kim CS, et al: Licochalcone-A induces intrinsic and extrinsic apoptosis via ERK1/2 and p38 phosphorylation-mediated TRAIL expression in head and neck squamous carcinoma FaDu cells. Food Chem Toxicol 77: 34-43, 2015.

8. Chi AC, Day TA and Neville BW: Oral cavity and oropharyngeal squamous cell carcinoma-an update. CA Cancer J Clin 65: 401-421, 2015.

9. Epstein JB, Thariat J, Bensadoun RJ, Barasch A, Murphy BA, Kolnick L, Popplewell L and Maghami E: Oral complications of cancer and cancer therapy: From cancer treatment to survivorship. CA Cancer J Clin 62: 400-422, 2012.

10. Fesik SW: Promoting apoptosis as a strategy for cancer drug discovery. Nat Rev Cancer 5: 876-885, 2005.

11. Shen H, Zeng G, Sun B, Cai X, Bi L, Tang G and Yang Y: A polysaccharide from Glycyrrhiza inflata Licorice inhibits proliferation of human oral cancer cells by inducing apoptosis via mitochondrial pathway. Tumour Biol 36: 4825-4831, 2015.

12. Lee CK, Park KK, Lim SS, Park JH and Chung WY: Effects of the licorice extract against tumor growth and cisplatin-induced toxicity in a mouse xenograft model of colon cancer. Biol Pharm Bull 30: 2191-2195, 2007.

13. Fu Y, Hsieh TC, Guo J, Kunicki J, Lee MY, Darzynkiewicz Z and $\mathrm{Wu} \mathrm{JM}$ : Licochalcone-A, a novel flavonoid isolated from licorice root (Glycyrrhiza glabra), causes G2 and late-G1 arrests in androgen-independent PC-3 prostate cancer cells. Biochem Biophys Res Commun 322: 263-270, 2004.

14. Jo EH, Kim SH, Ra JC, Kim SR, Cho SD, Jung JW, Yang SR, Park JS, Hwang JW, Aruoma OI, et al: Chemopreventive properties of the ethanol extract of Chinese licorice (Glycyrrhiza uralensis) root: Induction of apoptosis and G1 cell cycle arrest in MCF-7 human breast cancer cells. Cancer Lett 230: 239-247, 2005.

15. Cho JJ, Chae JI, Yoon G, Kim KH, Cho JH, Cho SS, Cho YS and Shim JH: Licochalcone A, a natural chalconoid isolated from Glycyrrhiza inflata root, induces apoptosis via Sp1 and Sp1 regulatory proteins in oral squamous cell carcinoma. Int J Oncol 45: 667-674, 2014.

16. Kinghorn AD, Pan L, Fletcher JN and Chai H: The relevance of higher plants in lead compound discovery programs. J Nat Prod 74: 1539-1555, 2011.

17. Wittschier N, Faller G and Hensel A: Aqueous extracts and polysaccharides from liquorice roots (Glycyrrhiza glabra L.) inhibit adhesion of Helicobacter pylori to human gastric mucosa. J Ethnopharmacol 125: 218-223, 2009.

18. Lee SK, Park KK, Park JH, Lim SS and Chung WY: The inhibitory effect of roasted licorice extract on human metastatic breast cancer cell-induced bone destruction. Phytother Res 27: 1776-1783, 2013.

19. Tamir S, Eizenberg M, Somjen D, Stern N, Shelach R, Kaye A and Vaya J: Estrogenic and antiproliferative properties of glabridin from licorice in human breast cancer cells. Cancer Res 60: 5704-5709, 2000.

20. Yoon G, Jung YD and Cheon SH: Cytotoxic allyl retrochalcone from the roots of Glycyrrhiza inflata. Chem Pharm Bull (Tokyo) 53: 694-695, 2005.

21. Lee HN, Cho HJ, Lim DY, Kang YH, Lee KW and Park JH: Mechanisms by which licochalcone e exhibits potent anti-inflammatory properties: Studies with phorbol ester-treated mouse skin and lipopolysaccharide-stimulated murine macrophages. Int J Mol Sci 14: 10926-10943, 2013. 
22. Zhou T, Deng X and Qiu J: Antimicrobial activity of licochalcone E against Staphylococcus aureus and its impact on the production of staphylococcal alpha-toxin. J Microbiol Biotechnol 22: 800-805, 2012.

23. Kim SS, Lim J, Bang Y, Gal J, Lee SU, Cho YC, Yoon G, Kang BY, Cheon SH and Choi HJ: Licochalcone E activates Nrf2/antioxidant response element signaling pathway in both neuronal and microglial cells: Therapeutic relevance to neurodegenerative disease. J Nutr Biochem 23: 1314-1323, 2012.

24. Park HG, Bak EJ, Woo GH, Kim JM, Quan Z, Kim JM, Yoon HK, Cheon SH, Yoon G, Yoo YJ, et al: Licochalcone E has an antidiabetic effect. J Nutr Biochem 23: 759-767, 2012.

25. Kwon SJ, Park SY, Kwon GT, Lee KW, Kang YH, Choi MS, Yun JW, Jeon JH, Jun JG and Park JH: Licochalcone E present in licorice suppresses lung metastasis in the 4T1 mammary orthotopic cancer model. Cancer Prev Res (Phila) 6: 603-613, 2013.

26. International head and neck cancer epidemiology consortium: Update no. 19. Head Neck 37: 1401-1402, 2015.

27. Howren MB, Christensen AJ, Karnell LH and Funk GF: Psychological factors associated with head and neck cancer treatment and survivorship: Evidence and opportunities for behavioral medicine. J Consult Clin Psychol 81: 299-317, 2013.

28. Ohnishi S and Takeda H: Herbal medicines for the treatment of cancer chemotherapy-induced side effects. Front Pharmacol 6: $14,2015$.

29. Lee KH: Research and future trends in the pharmaceutical development of medicinal herbs from Chinese medicine. Public Health Nutr 3: 515-522, 2000.

30. Peng F, Du Q, Peng C, Wang N, Tang H, Xie X, Shen J and Chen J: A review: The pharmacology of isoliquiritigenin. Phytother Res 29: 969-977, 2015.

31. Song NR, Kim JE, Park JS, Kim JR, Kang H, Lee E, Kang YG, Son JE, Seo SG, Heo YS and Lee KW: Licochalcone A, a polyphenol present in licorice, suppresses UV-induced COX-2 expression by targeting PI3K, MEK1, and B-Raf. Int J Mol Sci 16: 4453-4470, 2015 .

32. Kuwajima H, Taneda Y, Chen WZ, Kawanishi T, Hori K, Taniyama T, Kobayashi M, Ren J and Kitagawa I: Variation of chemical constituents in processed licorice roots: Quantitative determination of saponin and flavonoid constituents in bark removed and roasted licorice roots. Yakugaku Zasshi 119 945-955, 1999 (In Japanese).

33. Stewart PM and Prescott SM: Can licorice lick colon cancer? J Clin Invest 119: 760-763, 2009.

34. Hu C, Liu H, Du J, Mo B, Qi H, Wang X, Ye S and Li Z: Estrogenic activities of extracts of Chinese licorice (Glycyrrhiza uralensis) root in MCF-7 breast cancer cells. J Steroid Biochem Mol Biol 113: 209-216, 2009.

35. Jo EH, Hong HD, Ahn NC, Jung JW, Yang SR, Park JS, Kim SH Lee YS and Kang KS: Modulations of the Bcl-2/Bax family were involved in the chemopreventive effects of licorice root (Glycyrrhiza uralensis Fisch) in MCF-7 human breast cancer cell. J Agric Food Chem 52: 1715-1719, 2004.
36. Zeng G, Shen H, Yang Y, Cai X and Xun W: Licochalcone A as a potent antitumor agent suppresses growth of human oral cancer SCC-25 cells in vitro via caspase-3 dependent pathways. Tumour Biol 35: 6549-6555, 2014.

37. Yuan X, Li T, Xiao E, Zhao H, Li Y, Fu S, Gan L, Wang Z, Zheng Q and Wang Z: Licochalcone B inhibits growth of bladder cancer cells by arresting cell cycle progression and inducing apoptosis. Food Chem Toxicol 65: 242-251, 2014.

38. Yuan X, Li D, Zhao H, Jiang J, Wang P, Ma X, Sun X and Zheng Q: Licochalcone A-induced human bladder cancer T24 cells apoptosis triggered by mitochondria dysfunction and endoplasmic reticulum stress. Biomed Res Int 2013: 474272, 2013.

39. Huang HC, Tsai LL, Tsai JP, Hsieh SC, Yang SF, Hsueh JT and Hsieh YH: Licochalcone A inhibits the migration and invasion of human lung cancer cells via inactivation of the Akt signaling pathway with downregulation of MMP-1/-3 expression. Tumour Biol 35: 12139-12149, 2014

40. Xiao XY, Hao M, Yang XY, Ba Q, Li M, Ni SJ, Wang LS and Du X: Licochalcone A inhibits growth of gastric cancer cells by arresting cell cycle progression and inducing apoptosis. Cancer Lett 302: 69-75, 2011.

41. Yo YT, Shieh GS, Hsu KF, Wu CL and Shiau AL: Licorice and licochalcone-A induce autophagy in $\mathrm{LNCaP}$ prostate cancer cells by suppression of Bcl-2 expression and the mTOR pathway. J Agric Food Chem 57: 8266-8273, 2009.

42. Chang HJ, Yoon G, Park JS, Kim MH, Baek MK, Kim NH, Shin BA, Ahn BW, Cheon SH and Jung YD: Induction of apoptosis by the licochalcone $\mathrm{E}$ in endothelial cells via modulation of NF-kappaB and Bcl-2 Family. Biol Pharm Bull 30: 2290-2293, 2007.

43. Oberhammer FA, Hochegger K, Fröschl G, Tiefenbacher R and Pavelka M: Chromatin condensation during apoptosis is accompanied by degradation of lamin $\mathrm{A}+\mathrm{B}$, without enhanced activation of cdc2 kinase. J Cell Biol 126: 827-837, 1994.

44. Weissmann C: Fas ligand and Fas: a death factor and its receptor. Jpn J Cancer Res 85: inside front cover, 1994.

45. Li Y, Wang H, Wang Z, Makhija S, Buchsbaum D, LoBuglio A, Kimberly R and Zhou T: Inducible resistance of tumor cells to tumor necrosis factor-related apoptosis-inducing ligand receptor 2 -mediated apoptosis by generation of a blockade at the death domain function. Cancer Res 66: 8520-8528, 2006.

46. Ikner A and Ashkenazi A: TWEAK induces apoptosis through a death-signaling complex comprising receptor-interacting protein 1 (RIP1), Fas-associated death domain (FADD) and caspase-8. J Biol Chem 286: 21546-21554, 2011.

47. Li H, Zhu H, Xu CJ and Yuan J: Cleavage of BID by caspase 8 mediates the mitochondrial damage in the Fas pathway of apoptosis. Cell 94: 491-501, 1998.

48. Fischer B, Coelho D, Dufour P, Bergerat JP, Denis JM, Gueulette $\mathrm{J}$ and Bischoff P: Caspase 8-mediated cleavage of the pro-apoptotic BCL-2 family member BID in p53-dependent apoptosis. Biochem Biophys Res Commun 306: 516-522, 2003. 